\title{
GEOMETRIC PROPERTIES OF THE FOUR PARAMETERS WRIGHT FUNCTION
}

\author{
SOURAV DAS AND KHALED MEHREZ
}

\begin{abstract}
In this paper, four parameters Wright function is considered. Certain geometric properties such as starlikeness, convexity, uniform convexity and close-to-convexity are discussed for this function. Further, certain geometric properties of normalized Bessel function of the first kind and two parameters Wright function are studied as a consequence. Interesting corollaries and examples are provided to support that these results are better than the existing ones and improve several results available in the literature.
\end{abstract}

\section{INTRODUCTION}

The Wright function

$$
W_{\alpha, \beta}(z)=\sum_{k=0}^{\infty} \frac{z^{k}}{k ! \Gamma(\alpha k+\beta)}, \beta, z \in \mathbb{C}, \alpha>-1
$$

was introduced by E. M. Wright [33] in connection with the asymptotic theory of partitions. The Wright function plays vital role in fractional calculus [13,22, the Mikusiśki operational calculus, integral transforms of the Hankel type and stochastic processes. For a historical overview regarding the Wright function and its applications we refer to [21, Appendix F]. Note that $W_{\alpha, \beta}(z)$ is an entire function of order $1 /(1+\alpha)$ and also known as the generalized Bessel function [4,22]. These functions generalize hypergeometric functions 1,2 .

The four parameters Wright function 13,16

$$
\mathcal{W}_{(\mu, a),(\nu, b)}(z)=\sum_{k=0}^{\infty} \frac{z^{k}}{\Gamma(a+k \mu) \Gamma(b+k \nu)}, \quad a, b \in \mathbb{C}, \quad \mu, \nu \in \mathbb{R},
$$

was studied by E. M. Wright for the case $\mu, \nu>0$ in 32. Further, he derived several properties of $\mathcal{W}_{(\mu, a),(\nu, b)}(z)$ for the case $b=\nu=1$ and $-1<\mu<0$ in 34]. It can be verified [16] that if $\mu+\nu>0$, then the infinite series expansion $\sqrt{1.2}$ of $\mathcal{W}_{(\mu, a),(\nu, b)}(z)$ converges absolutely for all $z \in \mathbb{C}$. It is well-known (see $[13)$ that $\mathcal{W}_{(\mu, a),(\nu, b)}(z)$ is an entire function for $a, b \in \mathbb{C}$ and $0<-\mu<\nu$.

Let us consider that $\mathcal{H}$ denotes the class of all analytic functions inside the unit disk $\mathbb{D}=\{z:|z|<1\}$. Suppose that $\mathcal{A}$ is the class of all functions $f \in \mathcal{H}$ which are normalized by $f(0)=f^{\prime}(0)-1=0$ such that

$$
f(z)=z+\sum_{k=2}^{\infty} a_{k} z^{k}, \quad z \in \mathbb{D} .
$$

A function $f \in \mathcal{A}$ is said to be a starlike function (with respect to the origin 0 ) in $\mathbb{D}$, if $f$ is univalent in $\mathbb{D}$ and $f(\mathbb{D})$ is a star-like domain with respect to 0 in $\mathbb{C}$. This class of starlike functions is denoted by $\mathcal{S}^{*}$. The analytic characterization of $\mathcal{S}^{*}$ is given 6 below:

$$
\Re\left(\frac{z f^{\prime}(z)}{f(z)}\right)>0 \forall z \in \mathbb{D} \Longleftrightarrow f \in \mathcal{S}^{*} .
$$

Let $\eta \in[0,1)$ and $z \in \mathbb{D}$. If $\Re\left(\frac{z f^{\prime}(z)}{f(z)}\right)>\eta$, then the function $f \in \mathcal{A}$ is said to be a starlike function of order $\eta$. We denote the class of starlike functions of order $\eta$ by $\mathcal{S}^{*}(\eta)$.

A function $f \in \mathcal{A}$ is said to be convex in $\mathbb{D}$ if $f$ is univalent in $\mathbb{D}$ and $f(\mathbb{D})$ is a convex domain in $\mathbb{C}$. We denote this class of convex functions by $\mathcal{K}$. This class can be analytically characterized as follows:

$$
\Re\left(1+\frac{z f^{\prime \prime}(z)}{f^{\prime}(z)}\right)>0, \forall z \in \mathbb{D} \Longleftrightarrow f \in \mathcal{K} .
$$

2010 Mathematics Subject Classification. 30C45, 30D15, 33C10.

Key words and phrases. Wright function, analytic function, univalent function, starlike function, close-to-convex function. 
A function $f(z) \in \mathcal{A}$ is said to be a convex function of order $\eta(0 \leq \eta<1)$, if

$$
\Re\left(1+\frac{z f^{\prime \prime}(z)}{f^{\prime}(z)}\right)>\eta, \quad z \in \mathbb{D} .
$$

This class is denoted by $\mathcal{K}(\eta)$. In particular, $\mathcal{K}=\mathcal{K}(0)$ and $\mathcal{S}^{*}=\mathcal{S}^{*}(0)$. It is well-known that $z f^{\prime}$ is starlike if and only if $f \in \mathcal{A}$ is convex. A function $f(z) \in \mathcal{A}$ is said to be close-to-convex in $\mathbb{D}$ if $\exists$ a starlike function $g(z)$ in $\mathbb{D}$ such that

$$
\Re\left(\frac{z f^{\prime}(z)}{g(z)}\right)>0, \quad z \in \mathbb{D} .
$$

The class of all close-to-convex functions is denoted by $\mathcal{C}$. It can be easily verified that $\mathcal{K} \subset \mathcal{S}^{*} \subset \mathcal{C}$. It is well-known that every close-to-convex function in $\mathbb{D}$ is also univalent in $\mathbb{D}$.

A function $f \in \mathcal{A}$ is said to be uniformly convex (starlike) if for every circular arc $\gamma$ contained in $\mathbb{D}$ with center $\zeta \in \mathbb{D}$ the image arc $f(\gamma)$ is convex (starlike w.r.t. the image $f(\zeta)$ ). The class of all uniformly convex (starlike) functions is denoted by $U C V(U S T)$ [27]. In 10,11], A. W. Goodman introduced these classes. Later, F. Rønning 27] introduced a new class of starlike functions $\mathcal{S}_{p}$ defined by

$$
\mathcal{S}_{p}:=\left\{f: f(z)=z F^{\prime}(z), F \in U C V\right\} .
$$

For further details on geometric properties of analytic functions we refer to $4,6,8,9,14,15,18,19,25$, and references cited therein.

Problems for investing geometric properties including starlikeness, closed-to-convexity, convexity or univalency of family of analytic functions in the $\mathbb{D}$ involving special functions have always been attracted by several researchers $[3,4,6,8,9,14,15,20,24,28,29$, .

We observe that $\mathcal{W}_{(\mu, a),(\nu, b)}(z) \notin \mathcal{A}$. For this reason, we consider the following normalization of $\mathcal{W}_{(\mu, a),(\nu, b)}(z)$ as follows

$$
\begin{aligned}
\mathbb{W}_{(\mu, a),(\nu, b)}(z) & =z \Gamma(a) \Gamma(b) \mathcal{W}_{(\mu, a),(\nu, b)}(z) \\
& =\sum_{k=0}^{\infty} \frac{\Gamma(a) \Gamma(b) z^{k+1}}{\Gamma(a+k \mu) \Gamma(b+k \nu)}, a, b \in \mathbb{C}, \mu, \nu \in \mathbb{R} \\
& =\sum_{k=0}^{\infty} \alpha_{k} z^{k+1},
\end{aligned}
$$

where

$$
\alpha_{k}=\frac{\Gamma(a) \Gamma(b)}{\Gamma(a+k \mu) \Gamma(b+k \nu)} .
$$

Although in 1.3$), a, b, z \in \mathbb{C}$, however in this work $a$ and $b$ are restricted to real valued and $z \in \mathbb{D}$.

For two functions $f$ and $g$, which are analytic in $\mathbb{D}$, we say that the function the function $f(z)$ is subordinate to $g(z)$ in $\mathbb{D}$, and write $f(z) \prec g(z)$ or $f \prec g(z \in \mathbb{D})$, if there exists a function $w(z)$, which is analytic in $\mathbb{D}$ with $w(0)=0$ and $|w(z)|<1$ for all $z \in \mathbb{D}$, such that $f(z)=g(w(z))$, $z \in \mathbb{D}$. It is well-known that if $f(z) \prec g(z)(z \in \mathbb{D})$, then $f(0)=g(0)$ and $f(\mathbb{D}) \subset g(\mathbb{D})$. Furthermore, if the function $g(z)$ is univalent in $\mathbb{D}$, then $f(z) \prec g(z)$ if and only if $f(0)=g(0)$ and $f(\mathbb{D}) \subset g(\mathbb{D})$. Following definition will be very helpful to prove some of the main results.

Definition 1.1. Let $\left\{\beta_{n}\right\}_{n \geq 1}$ be a sequence of complex numbers. Then $\left\{\beta_{n}\right\}_{n \geq 1}$ is called a subordinating factor sequence, if

$$
f(z)=\sum_{n=1}^{\infty} \alpha_{n} z^{n} \in \mathcal{K}
$$

implies

$$
\sum_{n=1}^{\infty} \alpha_{n} \beta_{n} z^{n} \prec f(z) .
$$

This class is denoted by $\mathcal{F}$. A finite sequence $\left\{\beta_{n}\right\}_{n=1}^{k}$ is called a subordinating factor sequence if (1.4) yields 1.5 whenever $\alpha_{k+1}=\alpha_{k+2}=\cdots=0$. This class of such finite sequences of length $k$ is denoted by $\mathcal{F}_{k}$. 
This paper is organized as follows. We provide some lemmas in Section 2, which will be helpful to prove the main results. In Section 3, starlikeness, convexity and uniform convexity of $\mathcal{W}_{(\mu, a),(\nu, b)}(z)$, are discussed using the properties of Fox-Wright function. In Section 4, we provide some alternative conditions for starlikeness, convexity and uniform convexity of $\mathbb{W}_{(\mu, a),(\nu, b)}(z)$, which will be helpful to discuss close-to-convexity (univalency) of $\mathbb{W}_{(\mu, a),(\nu, b)}(z)$. In Section 5, we derive some properties and inequalities related to $\mathcal{W}_{(\mu, a),(\nu, b)}(z)$ and $\mathbb{W}_{(\mu, a),(\nu, b)}(z)$ involving hypergeometric function. In Section 6 , we discuss geometric properties of normalized Bessel function of the first kind and two parameters Wright function, as applications and show that the results obtained in this paper, are better than the existing ones available in the literature.

\section{Some Lemmas}

In this section, we provide some useful lemmas which will be useful to complete the proof of the main results.

Lemma 2.1 ( $[7])$. Let $\left\{a_{k}\right\}_{k=1}^{\infty}$ be a sequence of non-negative real numbers such that $a_{1}=1$. If $\left\{a_{k}\right\}_{k=2}^{\infty}$ is convex decreasing, i.e., $0 \geq a_{k+2}-a_{k+1} \geq a_{k+1}-a_{k}$, then

$$
\Re\left(\sum_{k=1}^{\infty} a_{k} z^{k-1}\right)>\frac{1}{2}, \quad z \in \mathbb{D} .
$$

Lemma 2.2 ([29] ). Let $\left\{\gamma_{k}\right\}$ be a sequence of complex numbers and $z \in \mathbb{D}$. Then the following statements are equivalent:

(i) $\left\{\gamma_{k}\right\}_{k=1}^{\infty} \in \mathcal{F}$.

(ii) $\Re\left(1+2 \sum_{k=1}^{\infty} \gamma_{k} z^{k}\right)>0$.

Lemma $2.3\left([17)\right.$. Let $f(z) \in \mathcal{A}$ and $\left|f^{\prime}(z)-1\right|<2 / \sqrt{5} \forall z \in \mathbb{D}$. Then $f(z)$ is a starlike function in $\mathbb{D}$.

Lemma 2.4 ( [14] $)$. Suppose that $f(z) \in \mathcal{A}$ and $|(f(z) / z)-1|<1 \forall z \in \mathbb{D}$. Then $f(z)$ is a univalent and starlike in $\mathbb{D}_{1 / 2}=\{z:|z|<1 / 2\}$.

Lemma 2.5 ( $[15)$. Let $f(z) \in \mathcal{A}$ and $\left|f^{\prime}(z)-1\right|<1 \forall z \in \mathbb{D}$. Then $f(z)$ is a convex function in $\mathbb{D}_{1 / 2}=\{z:|z|<1 / 2\}$.

Lemma $2.6(26)$. Let $f(z) \in \mathcal{A}$.

(i) If $\left|\frac{z f^{\prime \prime}(z)}{f^{\prime}(z)}\right|<\frac{1}{2}$, then $f(z) \in U C V$.

(ii) If $\left|\frac{z f^{\prime}(z)}{f(z)}-1\right|<\frac{1}{2}$, then $f(z) \in \mathcal{S}_{p}$.

Lemma 2.7. For any $a, b>0$, the following inequalities hold:

$$
\begin{array}{ll}
\frac{k}{a(a+1) \cdots(a+k-1)} \leq \frac{1}{a(a+1)^{k-2}}, & k \in \mathbb{N} \backslash\{1\}, \\
\frac{1}{b(b+1) \cdots(b+k-1)} \leq \frac{1}{b(b+1)^{k-1}}, \quad k \in \mathbb{N} .
\end{array}
$$

Proof. Under the given hypothesis, it can be observed that

$$
1\left(1+\frac{1}{a+1}\right)\left(1+\frac{2}{a+1}\right) \cdots\left(1+\frac{k-3}{a+1}\right)\left(1+\frac{a-1}{k}\right) \geq 1 .
$$

Multiplying both sides of $(2.8)$ by $a(a+1)^{k-2}$, we obtain

$$
a(a+1)(a+2) \cdots(a+k-2) \frac{(a+k-1)}{k} \geq a(a+1)^{k-2}, \quad \text { for } k \geq 2,
$$

which proves the inequality 2.6 .

It can be noted that under the given hypothesis, following inequality holds true:

$$
1\left(1+\frac{1}{b+1}\right)\left(1+\frac{2}{b+1}\right) \cdots\left(1+\frac{k-2}{b+1}\right) \geq 1 .
$$

Multiplying both sides of 2.9 by $b(b+1)^{k-1}$, we obtain

$$
b(b+1)(b+2) \cdots(b+k-1) \geq b(b+1)^{k-1}, \quad \text { for } k \geq 1,
$$


which proves the inequality 2.7 .

\section{StarlikenESs, CONVEXITY AND UNIFORM CONVEXITY}

To prove some of the main results, we need the Fox-Wright function ${ }_{p} \Psi_{q}[z]$, defined by 31 , p. 4, Eq. $(2.4)]$

$$
{ }_{p} \Psi_{q}\left[\begin{array}{c}
\left(a_{1}, A_{1}\right), \ldots,\left(a_{p}, A_{p}\right) \\
\left(b_{1}, B_{1}\right), \ldots,\left(b_{q}, B_{q}\right)
\end{array} \mid z\right]={ }_{p} \Psi_{q}\left[\begin{array}{c}
\left(a_{p}, A_{p}\right) \\
\left(b_{q}, B_{q}\right)
\end{array} \mid z\right]=\sum_{k=0}^{\infty} \frac{\prod_{i=1}^{p} \Gamma\left(a_{i}+k A_{i}\right)}{\prod_{j=1}^{q} \Gamma\left(b_{j}+k B_{j}\right)} \frac{z^{k}}{k !},
$$

where $A_{i}, B_{j} \in \mathbb{R}^{+}(i=1, \ldots, p, j=1, \ldots, q)$ and $a_{i}, b_{j} \in \mathbb{C}$. The series 3.10 converges uniformly and absolutely for all bounded $|z|, z \in \mathbb{C}$ when

$$
\epsilon=1+\sum_{j=1}^{q} B_{j}-\sum_{j=1}^{p} A_{j}>0
$$

In 23, Theorem 4], the authors established the following two-sided inequality

$$
\psi_{0} e^{\psi_{1} \psi_{0}^{-1}|z|} \leq{ }_{p} \Psi_{q}\left[\begin{array}{c}
\left(a_{p}, A_{p}\right) \\
\left(b_{q}, B_{q}\right)
\end{array} \mid z\right] \leq \psi_{0}-\left(1-e^{|z|}\right) \psi_{1},
$$

is valid for all $z \in \mathbb{R}$ and for all ${ }_{p} \Psi_{q}[z]$ satisfying

$$
\psi_{1}>\psi_{2} \text { and } \psi_{1}^{2}<\psi_{0} \psi_{2} \text {. }
$$

Here,

$$
\psi_{k}=\frac{\prod_{j=1}^{p} \Gamma\left(a_{j}+k A j\right)}{\prod_{j=1}^{q} \Gamma\left(b_{j}+k B_{j}\right)}, k=0,1,2
$$

Theorem 3.1. Assume that $a, b, \mu, \nu>0$. Suppose that the following conditions hold:

$$
\left(H_{1}\right): \begin{cases}(i) \quad \frac{\Gamma(a+2 \mu)}{\Gamma(a+3 \mu)}<\frac{\Gamma(b+3 \nu)}{3 \Gamma(b+2 \nu)} \\ \text { (ii) } \quad \frac{\Gamma(a+\mu) \Gamma(a+3 \mu)}{\Gamma^{2}(a+2 \mu)}<\frac{3 \Gamma^{2}(b+2 \nu)}{2 \Gamma(b+\nu) \Gamma(b+3 \nu)}, \\ (\text { iii }) \quad \frac{2 \Gamma(a) \Gamma(b)}{\Gamma(a+\mu) \Gamma(b+\nu)}+\frac{3(e-1) \Gamma(a) \Gamma(b)}{\Gamma(a+2 \mu) \Gamma(b+2 \nu)}<1 .\end{cases}
$$

Then the function $\mathbb{W}_{(\mu, a),(\nu, b)}(z)$ is starlike in $\mathbb{D}$.

Proof. Let us assume that $q(z)=\frac{z \mathbb{W}_{(\mu, a),(\nu, b)}^{\prime}(z)}{\mathbb{W}_{(\mu, a),(\nu, b)}(z)}, z \in \mathbb{D}$. Then $q(z)$ is analytic in $\mathbb{D}$ and $q(0)=1$. To prove that $\Re(q(z))>0$, it is enough to show that $|q(z)-1|<1$.

From 1.3 , we get

$$
\begin{aligned}
\left|\mathbb{W}_{(\mu, a),(\nu, b)}^{\prime}(z)-\frac{\mathbb{W}_{(\mu, a),(\nu, b)}(z)}{z}\right| & \leq \sum_{k=0}^{\infty} \frac{(k+1) \Gamma(a) \Gamma(b)}{\Gamma(a+\mu+k \mu) \Gamma(b+\nu+k \nu)} \\
& =\Gamma(a) \Gamma(b){ }_{1} \Psi_{2}\left[\begin{array}{c}
(2,1) \\
(a+\mu, \mu),(b+\nu, \nu)
\end{array} \mid 1\right] .
\end{aligned}
$$

In our case

$$
\psi_{0}=\frac{\Gamma(2)}{\Gamma(a+\mu) \Gamma(b+\nu)}, \psi_{1}=\frac{\Gamma(3)}{\Gamma(a+2 \mu) \Gamma(b+2 \nu)}, \text { and } \psi_{2}=\frac{\Gamma(4)}{\Gamma(a+3 \mu) \Gamma(b+3 \nu)} .
$$

It is easy to see that the hypotheses " $\left(H_{1}\right):(i),(i i)$ " are equivalent to $\psi_{2}<\psi_{1}$ and $\psi_{1}^{2}<\psi_{0} \psi_{2}$, and consequently

$$
{ }_{1} \Psi_{2}\left[\begin{array}{c|c}
(2,1) \\
(a+\mu, \mu),(b+\nu, \nu)
\end{array} \mid 1\right] \leq \frac{1}{\Gamma(a+\mu) \Gamma(b+\nu)}-\frac{2(1-e)}{\Gamma(a+2 \mu) \Gamma(b+2 \nu)} .
$$

Combining (3.13) and 3.14 , we obtain

$$
\left|\mathbb{W}_{(\mu, a),(\nu, b)}^{\prime}(z)-\frac{\mathbb{W}_{(\mu, a),(\nu, b)}(z)}{z}\right|<\frac{\Gamma(a) \Gamma(b)}{\Gamma(a+\mu) \Gamma(b+\nu)}-\frac{2(1-e) \Gamma(a) \Gamma(b)}{\Gamma(a+2 \mu) \Gamma(b+2 \nu)}, \text { for all } z \in \mathbb{D} .
$$


Now, using (1.3) and the triangle inequality $\left|z_{1}+z_{2}\right| \geq|| z_{1}|-| z_{2}||$, we have

$$
\begin{aligned}
& \left|\frac{\mathbb{W}_{(\mu, a),(\nu, b)}(z)}{z}\right| \geq 1-\left|\sum_{k=1}^{\infty} \frac{\Gamma(a) \Gamma(b) z^{k}}{\Gamma(a+k \mu) \Gamma(b+k \nu)}\right| \\
& \geq 1-\sum_{k=0}^{\infty} \frac{\Gamma(a) \Gamma(b)}{\Gamma(a+\mu+k \mu) \Gamma(b+\nu+k \nu)} \\
& =1-\Gamma(a) \Gamma(b) \sum_{k=0}^{\infty} \frac{\Gamma(k+1)}{\Gamma(a+\mu+k \mu) \Gamma(b+\nu+k \nu) k !} \\
& =1-\Gamma(a) \Gamma(b)_{1} \Psi_{2}\left[\begin{array}{c|c}
(1,1) & (a+\mu, \mu),(b+\nu, \nu)
\end{array} 1\right] .
\end{aligned}
$$

In this case, $\psi_{0}=\frac{1}{\Gamma(a+\mu) \Gamma(b+\nu)}, \psi_{1}=\frac{1}{\Gamma(a+2 \mu) \Gamma(b+2 \nu)}$ and $\psi_{2}=\frac{2}{\Gamma(a+3 \mu) \Gamma(b+3 \nu)}$. Clearly, the inequalities $\psi_{2}<\psi_{1}$ and $\psi_{1}^{2}<\psi_{0} \psi_{2}$ are satisfied under the given hypothesis. Using (3.11), we have

$$
{ }_{1} \Psi_{2}\left[\begin{array}{c|c}
(1,1) \\
(a+\mu, \mu),(b+\nu, \nu)
\end{array} \mid 1\right] \leq \frac{1}{\Gamma(a+\mu) \Gamma(b+\nu)}+\frac{(e-1)}{\Gamma(a+2 \mu) \Gamma(b+2 \nu)}
$$

Combining (3.15) and (3.16), we have

$$
\left|\frac{\mathbb{W}_{(\mu, a),(\nu, b)}(z)}{z}\right| \geq 1-\left[\frac{\Gamma(a) \Gamma(b)}{\Gamma(a+\mu) \Gamma(b+\nu)}+\frac{(e-1) \Gamma(a) \Gamma(b)}{\Gamma(a+2 \mu) \Gamma(b+2 \nu)}\right]>0,
$$

under the given hypothesis $\left(H_{1}:(\right.$ iii $\left.)\right)$. Now, using 3.13 and (3.17), we obtain

$$
\begin{aligned}
|q(z)-1| & =\left|\frac{z \mathbb{W}_{(\mu, a),(\nu, b)}^{\prime}(z)}{\mathbb{W}_{(\mu, a),(\nu, b)}(z)}-1\right|=\left|\frac{\mathbb{W}_{(\mu, a),(\nu, b)}^{\prime}(z)-\frac{\mathbb{W}_{(\mu, a),(\nu, b)}(z)}{z}}{\frac{\mathbb{W}_{(\mu, a),(\nu, b)}(z)}{z}}\right| \\
& <\left\lfloor\frac{\Gamma(a) \Gamma(b)}{\Gamma(a+\mu) \Gamma(b+\nu)}+\frac{2(e-1) \Gamma(a) \Gamma(b)}{\Gamma(a+2 \mu) \Gamma(b+2 \nu)}\right]\left[1-\frac{\Gamma(a) \Gamma(b)}{\Gamma(a+\mu) \Gamma(b+\nu)}-\frac{(e-1) \Gamma(a) \Gamma(b)}{\Gamma(a+2 \mu) \Gamma(b+2 \nu)}\right]^{-1} \\
& <1
\end{aligned}
$$

under the given hypothesis. This proves the theorem.

Theorem 3.2. Let $a, b, \mu$ and $\nu$ be positive real numbers. Also suppose that the following conditions hold:

$$
\left(H_{2}\right): \begin{cases}(i) & \frac{\Gamma(a+2 \mu)}{\Gamma(a+3 \mu)}<\frac{3 \Gamma(b+3 \nu)}{8 \Gamma(b+2 \nu)}, \\ \text { (ii }) & \frac{\Gamma(a+\mu) \Gamma(a+3 \mu)}{\Gamma^{2}(a+2 \mu)}<\frac{16 \Gamma^{2}(b+2 \nu)}{9 \Gamma(b+\nu) \Gamma(b+3 \nu)}, \\ \text { (iii }) & \frac{2 \Gamma(a) \Gamma(b)}{\Gamma(a+\mu) \Gamma(b+\nu)}-\frac{3(1-e) \Gamma(a) \Gamma(b)}{\Gamma(a+2 \mu) \Gamma(b+2 \nu)}<1 .\end{cases}
$$

Then the function $\mathbb{W}_{(\mu, a),(\nu, b)}(z)$ is convex in $\mathbb{D}_{\frac{1}{2}}$.

Proof. Let $z \in \mathbb{D}$, then we get

$$
\begin{aligned}
& \left|\mathbb{W}_{(\mu, a),(\nu, b)}^{\prime}(z)-1\right| \leq \sum_{k=1}^{\infty} \frac{(k+1) \Gamma(a) \Gamma(b)}{\Gamma(a+\mu k) \Gamma(b+k \nu)} \\
& =\sum_{k=0}^{\infty} \frac{(k+2) k ! \Gamma(a) \Gamma(b)}{k ! \Gamma(a+\mu+\mu k) \Gamma(b+\nu+k \nu)} \\
& =\Gamma(a) \Gamma(b)_{2} \Psi_{3}\left[\begin{array}{c|c}
(1,1),(3,1) & (2,1),(a+\mu, \mu),(b+\nu, \nu)
\end{array} \mid 1 .\right.
\end{aligned}
$$

In this case,

$$
\psi_{0}=\frac{2}{\Gamma(a+\mu) \Gamma(b+\nu)}, \psi_{1}=\frac{3}{\Gamma(a+2 \mu) \Gamma(b+2 \nu)}, \text { and } \psi_{2}=\frac{8}{\Gamma(a+3 \mu) \Gamma(b+3 \nu)} .
$$

Hence, the conditions " $\left(H_{2}\right):(i),(i i)$ " imply that

$$
{ }_{2} \Psi_{3}\left[\begin{array}{c|c}
(1,1),(3,1) \\
(2,1),(a+\mu, \mu),(b+\nu, \nu)
\end{array} \mid 1\right] \leq \frac{2}{\Gamma(a+\mu) \Gamma(b+\nu)}-\frac{3(1-e)}{\Gamma(a+2 \mu) \Gamma(b+2 \nu)} .
$$


Hence, combining the hypotheses " $\left(H_{2}\right):(i i i) ",(3.18)$ and $(3.19)$, we have

$$
\left|\mathbb{W}_{(\mu, a),(\nu, b)}^{\prime}(z)-1\right|<1 \text {. }
$$

Therefore, the function $\mathbb{W}_{(\mu, a),(\nu, b)}(z)$ is convex on $\mathbb{D}_{\frac{1}{2}}$, by means of Lemma 2.5

Corollary 3.3. Let $a>\frac{2}{7}$. If $b>\frac{2 a+20}{7 a-2}$, then the function $\mathbb{W}_{(1, a),(1, b)}(z)$ is convex on $\mathbb{D}_{\frac{1}{2}}$.

Proof. Setting $\mu=\nu=1$ in Theorem 3.2, we observe that the condition " $\left(H_{2}\right)$ : $(i)$ " holds true for all $a, b>0$. Moreover, it is clear that the condition " $\left(H_{2}\right):(i i)$ " is equivalent to the following inequality

$$
b>\frac{2 a+20}{7 a-2}:=f_{1}(a), \text { when } a>\frac{2}{7} .
$$

Straightforward calculation yields that the assumption " $\left(H_{2}\right):($ iii $) "$ is equivalent to

$$
b>\frac{-\left(a^{2}-a-2\right)+\sqrt{\left(a^{2}-a-1\right)^{2}+4 a(a+1)(2 a-1+3 e)}}{2 a(a+1)}:=g_{1}(a) .
$$

By using that fact that the functions $f_{1}(a)$ and $g_{1}(a)$ are decreasing on $(2 / 7, \infty)$ such that

$$
\lim _{a \rightarrow\left(\frac{2}{7}\right)^{+}} f_{1}(a)=\infty, \lim _{a \rightarrow \infty} f_{1}(a)=\frac{2}{7}, \lim _{a \rightarrow\left(\frac{2}{7}\right)^{+}} g_{1}(a) \approx 9.63 \text { and } \lim _{a \rightarrow \infty} g_{1}(a)=0 .
$$

Therefore

$$
b>\max _{a>\frac{2}{7}}\left(f_{1}(a), g_{1}(a)\right)=f_{1}(a) .
$$

Putting $a=14$ in Corollary 3.3 , we obtain the following example.

Example 3.4. If $b>\frac{1}{2}$, then the function $\mathbb{W}_{(1,14),(1, b)}(z)$ is convex on $\mathbb{D}_{\frac{1}{2}}$.

Theorem 3.5. Assume that the conditions of the above Theorem $\left(\mathrm{H}_{2}\right):(i),(i i)$ hold true. Also, suppose that

$$
\frac{2 \Gamma(a) \Gamma(b)}{\Gamma(a+\mu) \Gamma(b+\nu)}-\frac{3(1-e) \Gamma(a) \Gamma(b)}{\Gamma(a+2 \mu) \Gamma(b+2 \nu)}<\frac{2}{\sqrt{5}} .
$$

Then the function $\mathbb{W}_{(\mu, a),(\nu, b)}(z)$ is starlike in $\mathbb{D}$.

Proof. The proof is similar to the proof of the above Theorem 3.2 when we used Lemma 2.3 we omit the details.

Theorem 3.6. Suppose that $a, b, \mu$ and $\nu$ are positive real numbers. Assume that the following conditions hold:

$$
\left(H_{3}\right): \begin{cases}(i) & \frac{\Gamma(a+2 \mu)}{\Gamma(a+3 \mu)}<\frac{\Gamma(b+3 \nu)}{2 \Gamma(b+2 \nu)} \\ (i i) \quad \frac{\Gamma(a+\mu) \Gamma(a+3 \mu)}{\Gamma^{2}(a+2 \mu)}<\frac{2 \Gamma^{2}(b+2 \nu)}{\Gamma(b+\nu) \Gamma(b+3 \nu)} \\ (\text { iii }) \quad \frac{\Gamma(a) \Gamma(b)}{\Gamma(a+\mu) \Gamma(b+\nu)}-\frac{(1-e) \Gamma(a) \Gamma(b)}{\Gamma(a+2 \mu) \Gamma(b+2 \nu)}<1 .\end{cases}
$$

Then the function $\mathbb{W}_{(\mu, a),(\nu, b)}(z)$ is starlike in $\mathbb{D}_{\frac{1}{2}}$.

Proof. It can be verified that

$$
\begin{aligned}
\left|\frac{\mathbb{W}_{(\mu, a),(\nu, b)}(z)}{z}-1\right| & \leq \sum_{k=0}^{\infty} \frac{\Gamma(a) \Gamma(b)}{\Gamma(a+\mu+k \mu) \Gamma(b+\nu+k \nu)} \\
& =\Gamma(a) \Gamma(b)_{1} \Psi_{2}\left[\begin{array}{c}
(1,1) \\
(a+\mu, \mu),(b+\nu, \nu)
\end{array} \mid 1\right] .
\end{aligned}
$$

In this case,

$$
\psi_{0}=\frac{1}{\Gamma(a+\mu) \Gamma(b+\nu)}, \psi_{1}=\frac{1}{\Gamma(a+2 \mu) \Gamma(b+2 \nu)}, \text { and } \psi_{2}=\frac{2}{\Gamma(a+3 \mu) \Gamma(b+3 \nu)},
$$

which is equivalent to " $\left(H_{3}\right):(i),(i i)$ ". This implies that

$$
{ }_{1} \Psi_{2}\left[\begin{array}{c|c}
(1,1) \\
(a+\mu, \mu),(b+\nu, \nu)
\end{array} \mid 1\right] \leq \frac{1}{\Gamma(a+\mu) \Gamma(b+\nu)}-\frac{(1-e)}{\Gamma(a+2 \mu) \Gamma(b+2 \nu)} .
$$


Using the above inequality (3.20) and hypothesis " $\left(H_{3}\right):($ iii $)$ ", we obtain

$$
\left|\frac{\mathbb{W}_{(\mu, a),(\nu, b)}(z)}{z}-1\right|<1
$$

for all $z \in \mathbb{D}$, which implies that the function $\mathbb{W}_{(\mu, a),(\nu, b)}(z)$ is starlike in $\mathbb{D}_{\frac{1}{2}}$, by Lemma 2.4 .

Corollary 3.7. Let $a, b>0$. If $a b>2$, then $\mathbb{W}_{(1, a),(1, b)}(z)$ is starlike in $\mathbb{D}_{\frac{1}{2}}$.

Proof. Set $\mu=\nu=1$ in Theorem 3.6. Then the condition " $\left(H_{3}\right):(i)$ " is valid for each $a, b>0$ and the assumption " $\left(H_{3}\right):($ ii $)$ " holds true for all $a b>2$. Further, the hypothesis " $\left(H_{3}\right):($ iii $)$ " is equivalent to the following inequality:

Consequently,

$$
b>\frac{1-a^{2}+\sqrt{\left(a^{2}-1\right)^{2}+4(a+e)\left(a^{2}+a\right)}}{2 a(a+1)} .
$$

$$
b>\max _{a>0}\left(\frac{2}{a}, \frac{1-a^{2}+\sqrt{\left(a^{2}-1\right)^{2}+4(a+e)\left(a^{2}+a\right)}}{2 a(a+1)}\right)=\frac{2}{a} .
$$

Example 3.8. If $b>2$, then the function $\mathbb{W}_{(1,1),(1, b)}(z)$ is starlike in $\mathbb{D}_{\frac{1}{2}}$.

Example 3.9. The function $\mathbb{W}_{(1, \sqrt{2}),(1, \sqrt{3})}(z)$ is starlike in $\mathbb{D}_{\frac{1}{2}}$.

Theorem 3.10. Let $a, b, \nu$ and $\mu$ be positive real numbers and $z \in \mathbb{D}$. If the following conditions hold

$$
\left(H_{4}\right): \begin{cases}(i) & \frac{\Gamma(a+\mu)}{\Gamma(a+2 \mu)}<\frac{\Gamma(b+2 \nu)}{4 \Gamma(b+\nu)} \\ \text { (ii }) & \frac{\Gamma(a) \Gamma(a+2 \mu)}{\Gamma^{2}(a+\mu)}<\frac{4 \Gamma^{2}(b+2 \nu)}{3 \Gamma(b+\nu) \Gamma(b+3 \nu)} \\ (\text { iii }) & \frac{\Gamma(a) \Gamma(b)}{\Gamma(a+\mu) \Gamma(b+\nu)}-\frac{3(1-e) \Gamma(a) \Gamma(b)}{\Gamma(a+2 \mu) \Gamma(b+2 \nu)}<\frac{1}{4}\end{cases}
$$

then the function $\mathbb{W}_{(\mu, a),(\nu, b)}(z) \in U C V$.

Proof. Let $z \in \mathbb{D}$. It is easy to see that

$$
\begin{aligned}
\left|z \mathbb{W}_{(\mu, a),(\nu, b)}^{\prime \prime}(z)\right| & =\Gamma(a) \Gamma(b)\left|{ }_{1} \Psi_{2}\left[\begin{array}{c}
(3,1) \\
(a+\mu, \mu),(b+\nu, \nu)
\end{array} \mid z\right]\right| \\
& \leq \Gamma(a) \Gamma(b){ }_{1} \Psi_{2}\left[\begin{array}{c}
(3,1) \\
(a+\mu, \mu),(b+\nu, \nu)
\end{array} \mid 1\right] .
\end{aligned}
$$

Taking into consideration (3.11) and under the following assumptions

$$
\left(H_{4}^{\prime}\right): \frac{\Gamma(a+\mu)}{\Gamma(a+2 \mu)}<\frac{\Gamma(b+2 \nu)}{4 \Gamma(b+\nu)} \text { and } \frac{\Gamma(a+\mu) \Gamma(a+3 \mu)}{\Gamma^{2}(a+2 \mu)}<\frac{4 \Gamma^{2}(b+2 \nu)}{3 \Gamma(b+\nu) \Gamma(b+3 \nu)}
$$

we get

$$
\left|z \mathbb{W}_{(\mu, a),(\nu, b)}^{\prime \prime}(z)\right| \leq \frac{2 \Gamma(a) \Gamma(b)}{\Gamma(a+\mu) \Gamma(b+\nu)}-\frac{6(1-e) \Gamma(a) \Gamma(b)}{\Gamma(a+2 \mu) \Gamma(b+2 \nu)}
$$

However, for all $z \in \mathbb{D}$, we have

$$
\begin{aligned}
\left|\mathbb{W}_{(\mu, a),(\nu, b)}^{\prime}(z)\right| & =\left|1+\sum_{k=1}^{\infty} \frac{(k+1) \Gamma(a) \Gamma(b) z^{k}}{\Gamma(a+k \mu) \Gamma(b+k \nu)}\right| \\
& \geq 1-\sum_{k=1}^{\infty} \frac{(k+1) \Gamma(a) \Gamma(b) z^{k}}{\Gamma(a+k \mu) \Gamma(b+k \nu)} \\
& =1-\sum_{k=0}^{\infty} \frac{(k+2) \Gamma(a) \Gamma(b)}{\Gamma(a+\mu+k \mu) \Gamma(b+\nu+k \nu)} \\
& =1-\Gamma(a) \Gamma(b)_{2} \Psi_{3}\left[\begin{array}{c}
(1,1),(3,1) \\
(2,1),(a+\mu, \mu),(b+\nu, \nu)
\end{array} \mid 1\right] .
\end{aligned}
$$

Using 3.11, under the following assumptions

$$
\left(H_{4}^{\prime \prime}\right): \frac{\Gamma(a+2 \mu)}{\Gamma(a+3 \mu)}<\frac{3 \Gamma(b+3 \nu)}{8 \Gamma(b+2 \nu)} \text { and } \frac{\Gamma(a+\mu) \Gamma(a+3 \mu)}{\Gamma^{2}(a+2 \mu)}<\frac{16 \Gamma^{2}(b+2 \nu)}{9 \Gamma(b+\nu) \Gamma(b+3 \nu)},
$$


we obtain

$$
{ }_{2} \Psi_{3}\left[\begin{array}{l}
(1,1),(3,1) \\
(2,1),(a+\mu, \mu),(b+\nu, \nu)
\end{array} \mid 1\right] \leq \frac{2}{\Gamma(a+\mu) \Gamma(b+\nu)}+\frac{3(e-1)}{\Gamma(a+2 \mu) \Gamma(b+2 \nu)} .
$$

In view of the above inequality and 3.22 , we have

$$
\left|\mathbb{W}_{(\mu, a),(\nu, b)}^{\prime}(z)\right| \geq 1-\frac{2 \Gamma(a) \Gamma(b)}{\Gamma(a+\mu) \Gamma(b+\nu)}+\frac{3(e-1) \Gamma(a) \Gamma(b)}{\Gamma(a+2 \mu) \Gamma(b+2 \nu)} .
$$

We notice that the right hand side of the above inequality is positive under the assumption $\left(H_{4}\right):(i i i)$. Now, combining (3.21), (3.23) and " $\left(H_{4}\right):($ iii $)$ ", we get

$$
\left|\frac{\mathbb{W}_{(\mu, a),(\nu, b)}^{\prime \prime}(z)}{\mathbb{W}_{(\mu, a),(\nu, b)}^{\prime}(z)}\right|<1 \text { for all } z \in \mathbb{D} \text {. }
$$

Hence, the first assertions of Lemma 2.6 completes the proof of Theorem 3.10 .

Corollary 3.11. Let $a>3$. If $b>\frac{2 a+6}{a-3}$, then the function $\mathbb{W}_{(1, a),(1, b)}(z) \in U C V$.

Proof. Let $\mu=\nu=1$ in the above Theorem. Then the condition $\left(H_{4}\right):(i)$ " is valid for all $a b+a+b>3$. The second condition of $\left(H_{4}\right)$ is equivalent to $b(a-3)>6+2 a$. Finally, the condition " $\left(H_{4}\right):(i i i)$ " holds true for all

$$
b>\frac{-\left(a^{2}-3 a-4\right)+\sqrt{\left(a^{2}-3 a-4\right)^{2}+4(4 a+12 e-8)}}{2 a(a+1)} .
$$

Consequently,

$$
\begin{aligned}
b & >\max _{a>3}\left(\frac{2 a+6}{a-3}, \frac{-\left(a^{2}-3 a-4\right)+\sqrt{\left(a^{2}-3 a-4\right)^{2}+4(4 a+12 e-8)}}{2 a(a+1)}\right) \\
& =\frac{2 a+6}{a-3} .
\end{aligned}
$$

\section{Further Results on starlikeness, CONVEXity AND Close-to-COnVEXity}

In this section, we provide some alternative conditions for starlikeness, convexity and uniform convexity of $\mathbb{W}_{(\mu, a),(\nu, b)}(z)$, which will be useful to discuss close-to-convexity (univalency) of $\mathbb{W}_{(\mu, a),(\nu, b)}(z)$ in $\mathbb{D}$.

Theorem 4.1. Let $a, b, \mu, \nu \geq 1$ be such that $b \geq \phi(a)=\frac{3 a+2}{a(a+1)}$. Then

(i) $\mathbb{W}_{(\mu, a),(\nu, b)}(z)$ is starlike in $\mathbb{D}$.

(ii) $\mathbb{W}_{(\mu, a),(\nu, b)}(z)$ is close-to-convex with respect to the starlike function $\mathbb{W}_{(\mu, a),(1, b)}(z)$ in $\mathbb{D}$.

Proof. (i) Let $p(z)=\frac{z \mathbb{W}_{(\mu, a),(\nu, b)}^{\prime}(z)}{\mathbb{W}_{(\mu, a),(\nu, b)}(z)}, z \in \mathbb{D}$. Then $p(z)$ is analytic in $\mathbb{D}$ and $p(0)=1$. To prove that $\Re(p(z))>0$, it is enough to show that $|p(z)-1|<1$. It is well-known that

$$
\Gamma(a+k) \leq \Gamma(a+k \mu), \quad k \in \mathbb{N}, a, \mu>1 .
$$

Therefore,

$$
\frac{\Gamma(a)}{\Gamma(a+k \mu)} \leq \frac{\Gamma(a)}{\Gamma(a+k)}=\frac{1}{a(a+1) \cdots(a+k-1)}
$$

Similarly, we have

$$
\frac{\Gamma(b)}{\Gamma(b+k \nu)} \leq \frac{\Gamma(b)}{\Gamma(b+k)}=\frac{1}{b(b+1) \cdots(b+k-1)}
$$


With the help of Lemma 2.7 and the above inequalities 4.24 and 4.25 , we obtain

$$
\begin{aligned}
\left|\mathbb{W}_{(\mu, a),(\nu, b)}^{\prime}(z)-\frac{\mathbb{W}_{(\mu, a),(\nu, b)}(z)}{z}\right| & =\left|\sum_{k=1}^{\infty} \frac{k \Gamma(a) \Gamma(b) z^{k}}{\Gamma(a+k \mu) \Gamma(b+k \nu)}\right| \\
& \leq \sum_{k=1}^{\infty} \frac{k}{a b(a+1)(b+1) \cdots(a+k-1)(b+k-1)} \\
& <\frac{1}{a b}+\frac{1}{a b} \sum_{k=2}^{\infty} \frac{1}{(a+1)^{k-2}(b+1)^{k-1}} \\
& =\frac{1}{a b}+\frac{1}{a b(b+1)} \sum_{k=0}^{\infty}\left\{\frac{1}{(a+1)(b+1)}\right\}^{k} \\
& =\frac{(a+1)(b+1)+a}{a b\{(a+1)(b+1)-1\}}
\end{aligned}
$$

Again, we have

$$
\begin{aligned}
\left|\frac{\mathbb{W}_{(\mu, a),(\nu, b)}(z)}{z}\right| & \geq 1-\left|\sum_{k=1}^{\infty} \frac{\Gamma(a) \Gamma(b) z^{k}}{\Gamma(a+k \mu)(b+k \nu)}\right| \\
& \geq 1-\sum_{k=1}^{\infty} \frac{1}{a b(a+1)(b+1) \cdots(a+k-1)(b+k-1)} \\
& >1-\frac{1}{a b} \sum_{k=0}^{\infty}\left\{\frac{1}{(a+1)(b+1)}\right\}^{k}=1-\frac{(a+1)(b+1)}{a b(a b+a+b)} \\
& =\frac{a b(a+b+a b)-(a+1)(b+1)}{a b(a+b+a b)} .
\end{aligned}
$$

Therefore,

$$
\begin{aligned}
|p(z)-1| & =\left|\frac{z \mathbb{W}_{(\mu, a),(\nu, b)}^{\prime}(z)}{\mathbb{W}_{(\mu, a),(\nu, b)}(z)}-1\right|=\left|\frac{\mathbb{W}_{(\mu, a),(\nu, b)}^{\prime}(z)-\frac{\mathbb{W}_{(\mu, a),(\nu, b)}(z)}{z}}{\frac{\mathbb{W}_{(\mu, a),(\nu, b)}(z)}{z}}\right| \\
& <\frac{(a+1)(b+1)+a}{a b(a+b+a b)-(a+1)(b+1)} .
\end{aligned}
$$

Under the given condition, $\frac{(a+1)(b+1)+a}{a b(a+b+a b)-(a+1)(b+1)} \leq 1$. This shows that $\mathbb{W}_{(\mu, a),(\nu, b)}(z)$ is starlike in $\mathbb{D}$ and consequently the part (i) of this theorem is proved.

(ii) Now, we proceed to prove the part (ii). For this, we have to show that there exists a function $h \in \mathcal{S}^{*}$ such that

$$
\Re\left(\frac{z \mathbb{W}_{(\mu, a),(\nu, b)}^{\prime}(z)}{h(z)}\right)>0, \quad z \in \mathbb{D},
$$

which can be proved by showing that

$$
\left|\frac{z \mathbb{W}_{(\mu, a),(\nu, b)}^{\prime}(z)}{h(z)}-1\right|<1, \quad z \in \mathbb{D}
$$


Using part (i) of this Theorem 4.1, we can observe that $\mathbb{W}_{(\mu, a),(1, b)}(z)$ is starlike in $\mathbb{D}$ under the given hypothesis. Again using (2.6) and (2.7), we obtain

$$
\begin{aligned}
\left|\mathbb{W}_{(\mu, a),(\nu, b)}^{\prime}(z)-\frac{\mathbb{W}_{(\mu, a),(1, b)}(z)}{z}\right| & <\sum_{k=1}^{\infty}\left|\frac{(k+1) \Gamma(a) \Gamma(b)}{\Gamma(a+k \mu) \Gamma(b+k \nu)}-\frac{\Gamma(a) \Gamma(b)}{\Gamma(a+k) \Gamma(b+k)}\right| \\
& \leq \sum_{k=1}^{\infty}\left|\frac{k \Gamma(a) \Gamma(b)}{\Gamma(a+k) \Gamma(b+k)}\right| \\
& \leq \sum_{k=1}^{\infty} \frac{k}{a b(a+1)(b+1) \cdots(a+k-1)(b+k-1)} \\
& <\frac{1}{a b}+\frac{1}{a b} \sum_{k=2}^{\infty} \frac{1}{(a+1)^{k-2}(b+1)^{k-1}} \\
& =\frac{(a+1)(b+1)+a}{a b\{(a+1)(b+1)-1\}}
\end{aligned}
$$

and

$$
\begin{aligned}
\left|\frac{\mathbb{W}_{(\mu, a),(1, b)}(z)}{z}\right| & \geq 1-\left|\frac{\Gamma(a) \Gamma(b) z^{k}}{\Gamma(a+k) \Gamma(b+k)}\right| \\
& \geq 1-\sum_{k=1}^{\infty} \frac{1}{a b(a+1)(b+1) \cdots(a+k-1)(b+k-1)} \\
& >1-\frac{1}{a b} \sum_{k=0}^{\infty}\left\{\frac{1}{(a+1)(b+1)}\right\}^{k}=1-\frac{(a+1)(b+1)}{a b(a b+a+b)} \\
& =\frac{a b(a+b+a b)-(a+1)(b+1)}{a b(a+b+a b)} .
\end{aligned}
$$

Using the above inequalities and given conditions, we have

$$
\begin{aligned}
\left|\frac{z \mathbb{W}_{(\mu, a),(\nu, b)}^{\prime}(z)}{\mathbb{W}_{(\mu, a),(1, b)}(z)}-1\right| & =\left|\frac{\mathbb{W}_{(\mu, a),(\nu, b)}^{\prime}(z)-\frac{\mathbb{W}_{(\mu, a),(1, b)}(z)}{z}}{\frac{\mathbb{W}_{(\mu, a),(1, b)}(z)}{z}}\right| \\
& <\frac{(a+1)(b+1)+a}{a b(a+b+a b)-(a+1)(b+1)} \leq 1,
\end{aligned}
$$

which implies that $\Re\left(\frac{z \mathbb{W}_{(\mu, a),(\mu, b)}^{\prime}(z)}{\mathbb{W}_{(\mu, a),(1, b)}(z)}\right)>0$. Consequently, $\mathbb{W}_{(\mu, a),(\mu, b)}(z)$ is close-to-convex with respect to the starlike function $\mathbb{W}_{(\mu, a),(1, b)}(z)$ in $\mathbb{D}$, under the given hypothesis.

Theorem 4.2. Let $a, b, \mu, \nu \geq 1$ and $0 \leq \eta<1$ be such that $b \geq \psi(a, \eta)$, where

$$
\begin{aligned}
\psi(a, \eta) & =\frac{(a+1)+(1-\eta)\left(a+1-a^{2}\right)}{2 a(1-\eta)(a+1)} \\
& +\frac{\sqrt{\left\{(a+1)+(1-\eta)\left(a+1-a^{2}\right)\right\}^{2}-4 a(1-\eta)(a+1)\{(1-\eta)(a+1)+2 a+1\}}}{2 a(1-\eta)(a+1)} .
\end{aligned}
$$

Then $\mathbb{W}_{(\mu, a),(\nu, b)}(z) \in \mathcal{S}^{*}(\eta)$ for each $z \in \mathbb{D}$.

Proof. From the proof of Theorem 4.1, it can be observed that $\mathbb{W}_{(\mu, a),(\nu, b)}(z)$ is starlike function of order $\eta$, if $\frac{(a+1)(b+1)+a}{a b(a+b+a b)-(a+1)(b+1)} \leq 1-\eta$, which is a consequence of the given condition.

Using Lemma 2.6 and the similar technique as in Theorem 4.1, the following theorem can be obtained.

Theorem 4.3. Let $a, b, \mu, \nu \geq 1$ be such that $b \geq \tau(a)$, where

$$
\tau(a)=\frac{3(a+1)-a^{2}+\sqrt{a^{4}+14 a^{3}+35 a^{2}+30 a+9}}{2 a(a+1)} .
$$

Then $\mathbb{W}_{(\mu, a),(\nu, b)}(z) \in \mathcal{S}_{p}$ for each $z \in \mathbb{D}$. 
Theorem 4.4. Let $a, b, \mu, \nu \geq 1$. If $b \geq \phi_{1}(a)$, where

$$
\phi_{1}(a)=\frac{\left(a+1-a^{2}\right)+\sqrt{a^{4}+2 a^{3}+7 a^{2}+6 a+1}}{2 a(a+1)},
$$

then $\mathbb{W}_{(\mu, a),(\nu, b)}(z)$ is starlike in $\mathbb{D}_{1 / 2}$.

Proof. With the help of Lemma 2.7 and the inequalities 4.24 and 4.25 , we obtain

$$
\begin{aligned}
\left|\frac{\mathbb{W}_{(\mu, a),(\nu, b)}(z)}{z}-1\right| & <\sum_{k=1}^{\infty} \frac{1}{a b(a+1)(b+1) \cdots(a+k-1)(b+k-1)} \\
& \leq \frac{1}{a b} \sum_{k=0}^{\infty}\left\{\frac{1}{(a+1)(b+1)}\right\}^{k}=\frac{(a+1)(b+1)}{a b(a b+a+b)} .
\end{aligned}
$$

Using the given condition and Lemma 2.4 , we conclude that $\mathbb{W}_{(\mu, a),(\nu, b)}(z)$ is starlike in $\mathbb{D}_{1 / 2}$, under the given hypothesis.

Theorem 4.5. Let $a, b, \mu, \nu \geq 1$.

(i) If $b \geq \psi_{1}(a)$, where

$$
\psi_{1}(a)=\frac{(3-a)+\sqrt{a^{2}+2 a+9}}{2 a} .
$$

Then $\mathbb{W}_{(\mu, a),(\nu, b)}(z)$ is convex in $\mathbb{D}_{1 / 2}$.

(ii) If $b \geq \psi_{2}(a)$, where

$$
\psi_{2}(a)=\frac{\sqrt{5}(2 a+3)-2 a^{2}+\sqrt{4 a^{4}+8 \sqrt{5} a^{3}+20(1+\sqrt{5}) a^{2}+4(15+4 \sqrt{5}) a+45}}{4 a(a+1)} .
$$

Then $\mathbb{W}_{(\mu, a),(\nu, b)}(z)$ is starlike in $\mathbb{D}$.

Proof. Using inequalities 4.24 and 4.25 and Lemma 2.7, we have

$$
\begin{aligned}
\left|\mathbb{W}_{(\mu, a),(\nu, b)}^{\prime}(z)-1\right| & <\sum_{k=1}^{\infty} \frac{(k+1) \Gamma(a) \Gamma(b)}{\Gamma(a+k \mu) \Gamma(b+k \nu)} \\
\leq & \sum_{k=1}^{\infty} \frac{k+1}{a b(a+1)(b+1) \cdots(a+k-1)(b+k-1)} \\
= & \frac{1}{a b}+\sum_{k=2}^{\infty} \frac{k}{a b(a+1)(b+1) \cdots(a+k-1)(b+k-1)} \\
& +\sum_{k=1}^{\infty} \frac{1}{a b(a+1)(b+1) \cdots(a+k-1)(b+k-1)} \\
\leq & \frac{1}{a b}+\sum_{k=2}^{\infty} \frac{1}{a b(a+1)^{k-1}(b+1)^{k-2}}+\sum_{k=1}^{\infty} \frac{1}{a b(a+1)^{k-1}(b+1)^{k-1}} \\
= & \frac{1}{a b}+\frac{(a+2)}{a b(a+1)} \sum_{k=0}^{\infty}\left\{\frac{1}{(a+1)(b+1)}\right\}^{k} \\
= & \frac{2 a(b+1)+3 b+2}{a b\{(a+1)(b+1)-1\}} .
\end{aligned}
$$

Using Lemma 2.5 and given condition (i), we can conclude that $\mathbb{W}_{(\mu, a),(\nu, b)}(z)$ is convex in $\mathbb{D}_{1 / 2}$. Further using the given condition (ii) and with the help of Lemma 2.3 , it is easy to show that $\mathbb{W}_{(\mu, a),(\nu, b)}(z)$ is starlike in $\mathbb{D}$. Hence the theorem is proved.

If we set $a=\mu=\nu=1$ in the normalized four parameters Wright function (1.3), then we obtain the normalized form of a class of functions involving the confluent hypergeometric function as follows:

$$
\mathbb{F}(b, z)=z \sum_{k=0}^{\infty} \frac{\Gamma(b)}{\Gamma(b+k)} \frac{z^{k}}{k !}=z \times{ }_{0} F_{1}(-; b ; z),
$$

where ${ }_{0} F_{1}(-; b ; z)$ is the confluent hypergeometric function [1,2]. Following corollary provides a set of sufficient conditions for $\mathbb{F}(b, z)$ to be starlike and convex in the open unit disk $\mathbb{D}$ and in $\mathbb{D}_{1 / 2}$. 
Corollary 4.6. The following assertions hold true:

(i) If $b \geq \frac{5}{4}$, then $\mathbb{F}(b, z)$ is starlike in the open unit disk $\mathbb{D}$.

(ii) If $b \geq \frac{(5+\sqrt{89})}{4}$, then $\mathbb{F}(b, z) \in \mathcal{S}_{p}$.

(iii) If $b \geq \frac{(1+\sqrt{17})}{4}$, then $\mathbb{F}(b, z)$ is starlike in $\mathbb{D}_{1 / 2}$.

(iv) If $b \geq 1+\sqrt{3}$, then $\mathbb{F}(b, z)$ is convex on $\mathbb{D}_{1 / 2}$.

Proof. Part (i) can be proved using Theorem 4.1. Part (ii) is a consequence of Theorem 4.3. Using part (i) of Theorem 4.4 part (iii) of this corollary can be obtained. Finally using Theorem 4.5 part (iv) can be proved.

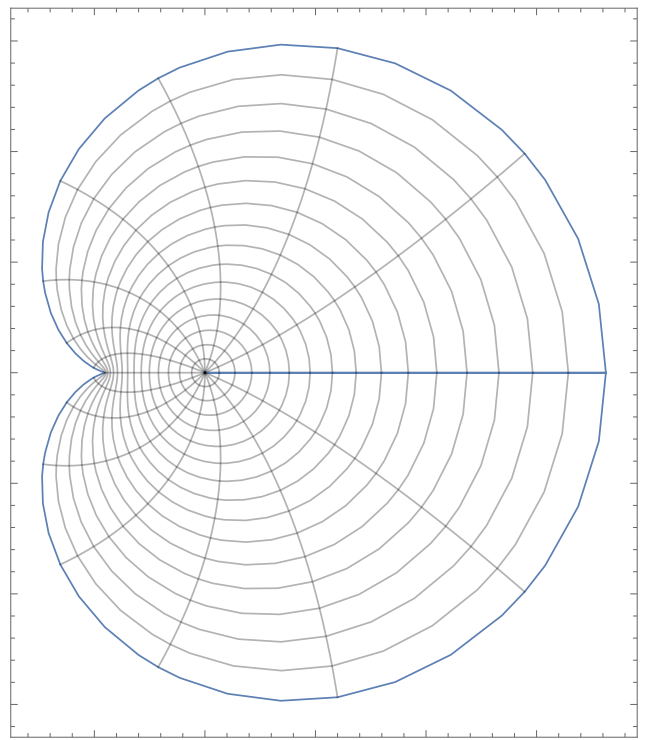

(A) $\mathbb{F}(3 / 2, z)$ for $z \in \mathbb{D}$.

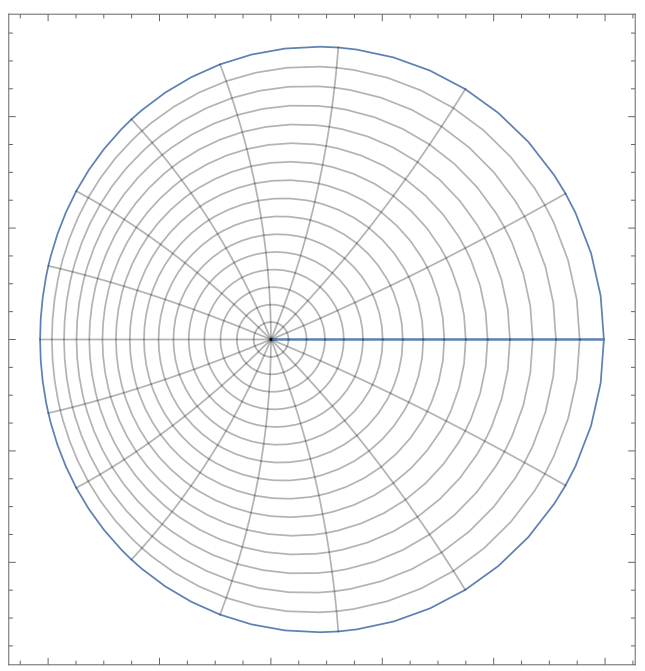

(C) $\mathbb{F}(1+\sqrt{3}, z)$ for $z \in \mathbb{D}_{1 / 2}$.

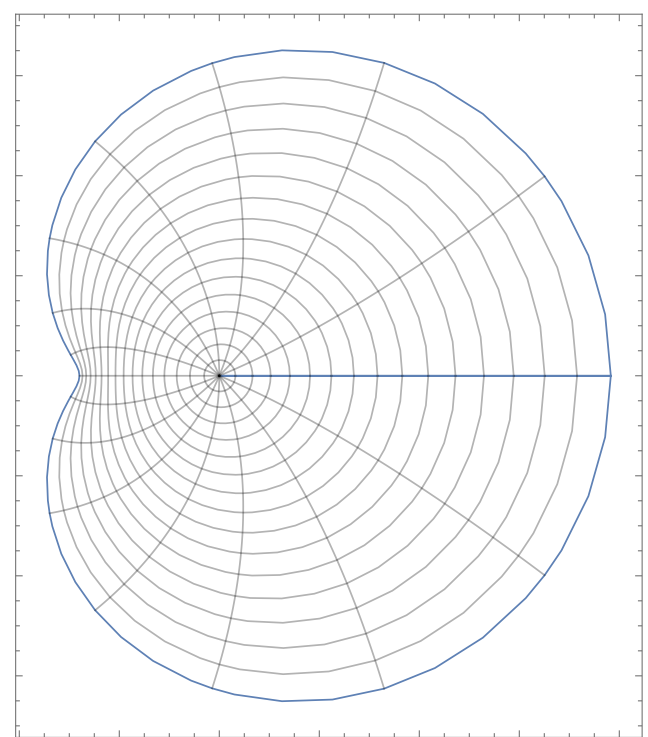

(B) $\mathbb{F}(1, z)$ for $z \in \mathbb{D}_{1 / 2}$.

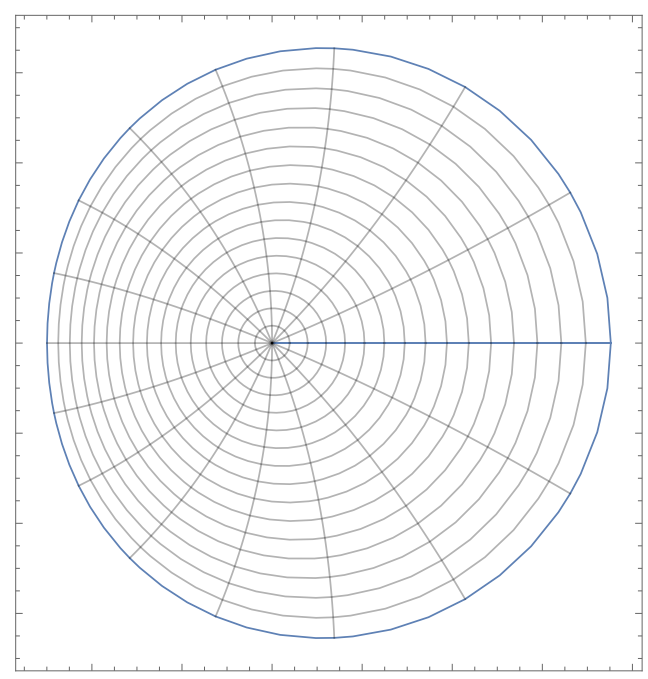

(D) $\mathbb{W}_{(1, \sqrt{2}),(1, \sqrt{3})}(z)$ for $z \in \mathbb{D}_{1 / 2}$.

Figure 1. Mapping of $\mathbb{F}(b, z)$ and $\mathbb{W}_{(\mu, a),(\nu, b)}(z)$ over $\mathbb{D}$ and $\mathbb{D}_{1 / 2}$.

5. Results Related to $\mathcal{W}_{(\mu, a),(\nu, b)}(z)$

Theorem 5.1. For any $a, b, \mu, \nu \geq 1$ and $z \in \mathbb{D}$, the following inequality holds:

$$
\left|\mathbb{W}_{(\mu, a),(\nu, b)}(z)\right| \leq|z| \times{ }_{1} F_{2}(1 ; a, b ;|z|),
$$


where ${ }_{1} F_{2}(c ; a, b ;|z|)=\sum_{k=0}^{\infty} \frac{(c)_{k}}{(a)_{k}(b)_{k}} \frac{|z|^{k}}{k !}$ is hypergeometric function with $(\alpha)_{k}$ as pochhammer symbol defined as $(\alpha)_{0}=1,(\alpha)_{k}=\alpha(\alpha+1) \cdots(\alpha+k-1)$.

Proof. By using 2.6 and (2.7), we obtain

$$
\begin{aligned}
\left|\mathbb{W}_{(\mu, a),(\nu, b)}(z)\right| & =\left|\sum_{k=0}^{\infty} \frac{\Gamma(a) \Gamma(b) z^{k+1}}{\Gamma(k \mu+a) \Gamma(k \nu+b)}\right| \\
& \leq|z| \sum_{k=0}^{\infty}\left|\frac{\Gamma(a) \Gamma(b) z^{k}}{\Gamma(k \mu+a) \Gamma(k \nu+b)}\right| \\
& \leq|z| \sum_{k=0}^{\infty} \frac{|z|^{k}}{a b(a+1)(b+1) \cdots(a+k-1)(b+k-1)} \\
& =|z| \sum_{k=0}^{\infty} \frac{(1)_{k}}{(a)_{k}(b)_{k}} \frac{|z|^{k}}{k !}=|z| \times{ }_{1} F_{2}(1 ; a, b ;|z|)
\end{aligned}
$$

Using (1.1) and Theorem 5.1, following corollary can be obtained.

Corollary 5.2. For any $a, b, \mu, \nu \geq 1$ and $z \in \mathbb{D}$ the following inequality holds:

$$
\left|\mathcal{W}_{(\mu, a),(\nu, b)}(z)\right| \leq \frac{|z|}{\Gamma(a) \Gamma(b)} \times{ }_{1} F_{2}(1 ; a, b ;|z|),
$$

where ${ }_{1} F_{2}(1 ; a, b ;|z|)$ is a hypergeometric function.

\section{Conclusion}

Our research discusses about some geometric properties (such as starlikeness, convexity, uniform convexity and close-to-convexity) of four parameters Wright function. In addition, geometric properties of the partial sums of this function are studied. As applications, we obtain certain geometric properties of normalized Bessel function of the first kind and two parameters Wright function as given below.

(1) It can be noted that for $a=\mu=\nu=1, b=\beta+1$ and $z=-z(z \in \mathbb{D})$, we have a normalization of the Bessel function of first kind [4] $\mathcal{J}_{\beta}(z)$ defined as [24]:

$$
\mathbb{J}_{\beta}(z)=\mathbb{W}_{(1, \beta+1),(1,1)}(-z)=\Gamma(\beta+1) z^{1-\beta / 2} \mathcal{J}_{\beta}(2 \sqrt{z}) .
$$

(i) Using Theorem 4.1, we claim that $\mathbb{J}_{\beta}(z)$ is starlike in $\mathbb{D}$ if $\beta \geq 3 / 2$, which is a sharper than the lower bound $(\sqrt{3})$ available in 24 .

(ii) Using Theorem 4.5 , we obtain $\mathbb{J}_{\beta}(z)$ is convex in $\mathbb{D}_{1 / 2}$ if $\beta \geq \sqrt{3}$, which is the same condition available in 24 .

(iii) Theorem 4.3 helps us to conclude that $\mathbb{J}_{\beta}(z) \in \mathcal{S}_{p}$ if $\beta \geq \frac{(1+\sqrt{89})}{4}$.

(2) For $a=\mu=1$, the four parameters Wright function reduces to two parameters Wright function

$$
W_{b, \nu}(z)=\mathcal{W}_{(1,1),(\nu, b)}(z)=\sum_{k=0}^{\infty} \frac{z^{k}}{k ! \Gamma(b+k \nu)},
$$

and the corresponding normalized two parameters Wright function can be defined as

$$
\mathbb{W}_{b, \nu}(z)=\mathbb{W}_{(1,1),(\nu, b)}(z)=\Gamma(b) W_{b, \nu}(z)=\sum_{k=0}^{\infty} \frac{\Gamma(b) z^{k}}{k ! \Gamma(b+k \nu)} .
$$

(i) Using Theorem 4.1, we claim that if $\nu \geq 1$ and $b \geq 5 / 2$, then $\mathbb{W}_{b, \nu}(z)$ is starlike in $\mathbb{D}$. This lower bound of $b$ is sharper than the lower bound $(b \geq 1+\sqrt{3})$ available in 24.

(ii) From Theorem 4.5 , we have if $\nu \geq 1$ and $b \geq(1+\sqrt{3})$, then $\mathbb{W}_{b, \nu}(z)$ is convex in $\mathbb{D}_{1 / 2}$, which is the same condition available in 24 .

(iii) From Theorem 4.1, we obtain, if $\nu \geq 1$ and $b \geq 5 / 2$, then $\mathbb{W}_{b, \nu}(z)$ is close-to-convex with respect to $\mathbb{W}_{1, \nu}(z)$ in $\mathbb{D}$.

(iv) Using Theorem 4.3 , we conclude that $\mathbb{W}_{b, \nu}(z) \in \mathcal{S}_{p}$ if $b \geq \frac{(5+\sqrt{89})}{4}$. 
(v) Setting $a=\mu=1$ and $b=4$ in Theorem 3.2 we obtain that $\mathbb{W}_{4, \nu}(z)$ is convex in $\mathbb{D}_{\frac{1}{2}}$ if $\nu \in[0.76,0.95]$. Putting $a=\mu=1$ and $b=2$ in Theorem 3.6. we have $\mathbb{W}_{2, \nu}(z)$ is starlike in $\mathbb{D}_{\frac{1}{2}}$ if $\nu \in[0.645,0.999]$. Similarly, we can verify that the other results obtained in Section 3 will be useful to discuss the geometric properties of $\mathbb{W}_{b, \nu}(z)$ when $0<\nu<1$. In literature, various results involving geometric properties of $\mathbb{W}_{b, \nu}(z)$ are available 24 ] with the condition that $b, \nu \geq 1$. But the results obtained in Section 3 discuss the case $0<\nu<1$. On the other hand, the results obtained in Section 4 , will be helpful to discuss the geometric properties of $\mathbb{W}_{b, \nu}(z)$ when $b, \nu \geq 1$.

\section{REFERENCES}

[1] M. Abramowitz and I. A. Stegun, Handbook of mathematical functions with formulas, graphs, and mathematical tables, National Bureau of Standards Applied Mathematics Series, 55, For sale by the Superintendent of Documents, U.S. Government Printing Office, Washington, DC, 1964.

[2] G. E. Andrews, R. Askey and R. Roy, Special functions, Encyclopedia of Mathematics and its Applications, 71, Cambridge University Press, Cambridge, 1999.

[3] D. Bansal and J. K. Prajapat, Certain geometric properties of the Mittag-Leffler functions, Complex Var. Elliptic Equ. 61 (2016), no. 3, 338-350.

[4] Á. Baricz, Generalized Bessel functions of the first kind, Lecture Notes in Mathematics, 1994, Springer-Verlag, Berlin, 2010.

[5] J. Bustoz, M.E.H. Ismail, On gamma function inequalities, Math. Comp. 47 (1986) 659-667.

[6] P. L. Duren, Univalent functions, Grundlehren der Mathematischen Wissenschaften, 259, Springer-Verlag, New York, 1983.

[7] L. Féjer, Untersuchungen ber Potenzreihen mit mehrfach monotoner Koeffizientenfolge. Acta iterarum Sci. 8 (1936), $89-115$.

[8] A. W. Goodman, Univalent functions. Vol. I, Mariner Publishing Co., Inc., Tampa, FL, 1983.

[9] A. W. Goodman, Univalent functions. Vol. II, Mariner Publishing Co., Inc., Tampa, FL, 1983.

[10] A. W. Goodman, On uniformly starlike functions, J. Math. Anal. Appl. 155 (1991), no. 2, 364-370.

[11] A. W. Goodman, On uniformly convex functions, Ann. Polon. Math. 56 (1991), no. 1, 87-92.

[12] S. Kakeya, On the limits of the roots of an algebraic equation with positive coefficients. Thoku Math. J. 2 (1912), 140-142.

[13] Y. Luchko and R. Gorenflo, Scale-invariant solutions of a partial differential equation of fractional order, Fract. Calc. Appl. Anal. 1 (1998), no. 1, 63-78.

[14] T. H. MacGregor, The radius of univalence of certain analytic functions. II, Proc Amer. Math. Soc. 14 (1963), $521-524$.

[15] T. H. MacGregor, A class of univalent functions, Proc. Amer. Math. Soc. 15 (1964), 311-317.

[16] K. Mehrez, New integral representations for the Fox-Wright functions and its applications, J. Math. Anal. Appl. 468 (2018), no. 2, 650-673.

[17] P. T. Mocanu, Some starlike conditions for analytic functions. Rev. Roumaine. Math. Pures. Appl. 33, (1988), 117-124.

[18] P. T. Mocanu, Some simple criteria for starlikeness and convexity, Libertas Math. 13 (1993), $27-40$.

[19] S. Noreen,M. Raza, J. -L. Liu 2, M. Arif, Geometric Properties of Normalized Mittag-Leffler Functions, Symmetry 2019, 11, 45.

[20] S. Ozaki, On the theory of multivalent functions, Sci. Rep. Tokyo Bunrika Daigaku A, 2 (1935), $167-188$.

[21] F. Mainardi, Fractional Calculus and Waves in Linear Viscoelasticity, Imperial College Press, London, 2010.

[22] I. Podlubny, Fractional differential equations, Mathematics in Science and Engineering, 198, Academic Press, Inc., San Diego, CA, 1999.

[23] T. K. Pogány and H. M. Srivastava, Some Mathieu-type series associated with the Fox-Wright function, Comput. Math. Appl. 57 (2009), no. 1, 127-140.

[24] J. K. Prajapat, Certain geometric properties of the Wright function, Integral Transforms Spec. Funct. 26 (2015), no. 3, $203-212$.

[25] R. K. Raina, On univalent and starlike Wright's hypergeometric functions, Rend. Sem. Mat. Univ. Padova 95 (1996), $11-22$.

[26] V. Ravichandran, On uniformly convex functions, Ganita 53 (2002), no. 2, 117-124.

[27] F. Rønning, Uniformly convex functions and a corresponding class of starlike functions, Proc. Amer. Math. Soc. 118 (1993), no. 1, 189-196.

[28] A. Swaminathan, Sufficient conditions for hypergeometric functions to be in a certain class of analytic functions, Comput. Math. Appl. 59 (2010), no. 4, 1578-1583.

[29] H. S. Wilf, Subordinating factor sequences for convex maps of the unit circle, Proc. Amer. Math. Soc. 12 (1961), 689-693.

[30] E. M. Wright, On the Coefficients of Power Series Having Exponential Singularities, J. London Math. Soc. 8 (1933), no. 1, 71-79.

[31] E. M. Wright, The Asymptotic Expansion of the Generalized Bessel Function, Proc. London Math. Soc. (2) 38 (1935), $257-270$.

[32] E. M. Wright, The asymptotic expansion of the generalized hypergeometric function, J. London Math. Soc. 10 (1935), $287-293$

[33] E. M. Wright, On the coefficients of power series having exponential singularities, Journal London Math. Soc. 8 (1933), 71-79. 
[34] E. M. Wright, The generalized Bessel function of order greater than one, Quart. J. Math., Oxford Ser. 11 (1940), $36-48$.

Sourav Das

Department of Mathematics, National Institute of Technology Jamshedpur, JAMSHEDPUR-831014, JHARKHAND, INDIA.

Email address: souravdasmath@gmail.com, souravdas.math@nitjsr.ac.in

KhaLed MehreZ

Département de Mathématiques, Faculté de Sciences de Tunis,

Université Tunis El Manar, Tunisia.

Email address: k.mehrez@yahoo.fr 